\title{
Long-term outcome of large sylvian arachnoid cysts: the role of surgery has been exaggerated
}

\author{
Kyung Hyun Kim, MD,1 Ji Yeoun Lee, MD, PhD,,2 Ji Hoon Phi, MD, PhD, ${ }^{1}$ Seung-Ki Kim, MD, PhD,1 \\ Byung-Kyu Cho, MD, PhD, ${ }^{3}$ and Kyu-Chang Wang, MD, PhD ${ }^{1}$
}

'Division of Pediatric Neurosurgery, Seoul National University Children's Hospital, Seoul; '2Department of Anatomy and Cell Biology, Seoul National University College of Medicine, Seoul; and ${ }^{3}$ Department of Neurosurgery, Armed Forces Capital Hospital, Seongnam, Republic of Korea

OBJECTIVE The surgical indications for some arachnoid cysts (ACs) are controversial. While surgical procedures can be effective when an $A C$ is a definite cause of hydrocephalus or papilledema, most ACs do not cause any symptoms or signs. Some surgeons perform several procedures to treat ACs because of their large size. The purpose of this study was to compare the long-term outcomes of Galassi type III ACs between surgery and nonsurgery groups.

METHODS The medical records of 60 patients diagnosed with sylvian ACs (Galassi type III) who visited Seoul National University Children's Hospital from July 1990 to March 2018 were analyzed. The authors compared the outcomes between those treated with surgery and those not treated with surgery.

RESULTS Of the 60 patients, 27 patients had no symptoms, 19 patients had vague symptoms and signs associated with ACs, and the remaining 14 patients had definite AC-related symptoms and signs. Thirty-eight patients underwent surgery, and 22 patients underwent observation. Some operations were accompanied by complications. Among the 33 patients in the surgery group, excluding 5 with hydrocephalus or papilledema, 8 patients needed 18 additional operations. However, there were no patients in the nonsurgery group who needed surgical intervention during the follow-up period (mean 67.5 months), although the size of the AC increased in 2 patients. Changes in AC size were not correlated with symptom relief.

CONCLUSIONS When patients with hydrocephalus or papilledema were excluded, there was no difference in the outcomes between the surgery and nonsurgery groups regardless of the size of the sylvian AC. Surgeons should be cautious when deciding whether to operate.

https://thejns.org/doi/abs/10.3171/2020.3.PEDS19574

KEYWORDS arachnoid cyst; children; sylvian fissure; surgery; observation; congenital

$\mathrm{A}$ RACHNOID cysts (ACs) are benign cystic lesions that are formed by a split of the arachnoid membrane and are filled with CSF. ACs are more often found in children than adults. Most ACs are located in the supratentorial and middle cranial fossae. Middle cranial fossa (sylvian fissure) ACs were classified by Galassi et al. according to their size in 1982. ${ }^{1}$ Some researchers argue that there are several indications for AC surgery. We agree that surgery is appropriate in cases with coexisting hydrocephalus or documented increased intracranial pressure (ICP), such as papilledema. However, in some studies, AC surgeries were performed just because of their large size. Our group does not actively recommend surgery if the patient does not have associated hydrocephalus or papilledema and the parents prefer conservative management. Our cumulative experience with the benign course of unoperated large sylvian AC cases made us evaluate the value of surgery in large sylvian ACs. This study was designed to compare the outcomes of the surgery group with those of the nonsurgery group for large sylvian ACs (Galassi type III).

\section{Methods \\ Patients}

This study was approved by the institutional review board of the Seoul National University Hospital.

We reviewed the medical records of 66 patients with

ABBREVIATIONS $\mathrm{AC}=$ arachnoid cyst; $\mathrm{CP}=$ cystoperitoneal; $\mathrm{ICP}=$ intracranial pressure; $\mathrm{SDFC}=$ subdural fluid collection; $\mathrm{SDH}=$ subdural hematoma .

SUBMITTED October 3, 2019. ACCEPTED March 6, 2020.

INCLUDE WHEN CITING Published online May 15, 2020; DOI: 10.3171/2020.3.PEDS19574. 
large sylvian ACs (Galassi type III) who visited the Division of Pediatric Neurosurgery, Seoul National University Children's Hospital, from July 1990 to March 2018. Among them, 6 patients with a follow-up duration of less than 6 months were excluded. The surgical procedures were performed by 4 experienced pediatric neurosurgeons.

Patients were divided into 2 groups: surgery and nonsurgery. Then, they were divided into 3 subgroups based on the chief complaint of the patient or the reason for the CT scan or MR image.

ACs with hydrocephalus or signs of increased ICP (papilledema) were absolute indications for surgery. Five patients, 4 with hydrocephalus and 1 with papilledema, were included in the study. In addition, if midline shift, focal bony bulging, or macrocephaly was present, the patients were operated on or observed regardless of the symptoms, depending on the decision of the surgeons and/ or guardians. As experience has accumulated over time, the patients have been increasingly managed with observation without surgery. Shunting $(n=5)$ was used as a surgical method until the 1990s, but endoscopic fenestration $(\mathrm{n}=9)$ and microsurgical fenestration $(\mathrm{n}=24)$ became the preferred methods thereafter.

Clinical presentations were classified as incidental, vague, or definite AC-related symptoms and signs. Incidental presentations included a fall, traffic accident, foreign body ingestion, and antenatal sonography. Vague symptoms and signs included headache or gaze palsy not typical of increased ICP, seizure, precocious puberty, and developmental delay. Definite AC-related symptoms and signs included focal bony bulging, macrocephaly, and papilledema.

\section{Clinical Data}

We assessed the patient's age, sex, reasons (symptoms or signs) for imaging, laterality of the $\mathrm{AC}$, extent of the $\mathrm{AC}$, coexisting hydrocephalus, type of operations, change in size, clinical outcomes, and complications (newly developed neurological deficit, seizure, subdural fluid collection (SDFC), subdural hematoma (SDH), infection, CSF leakage, and CSF overdrainage) associated with surgical treatment.

The size of the ACs was measured using CT or MRI on the first and most recent scans. The average intervals between the first and the most recent images of nonsurgery and surgery groups were 67 months and 105 months, respectively. The patients were followed up with neuroimaging at least until the age of 3 years in the nonsurgery group. Our previous study showed that the size of the AC remained stable after 3 years of age. ${ }^{2}$

For the description of size change, "increase" was defined as $\geq 10 \%$ enlargement. An enlargement of $<10 \%$ or a reduction of $<30 \%$ was defined as no change. A reduction of $\geq 30 \%$ and $<50 \%$ was defined as a "slight decrease," and a $\geq 50 \%$ reduction was defined as a "definite decrease." Volumes were measured by a planimetric method and the simplified formula for the volume of an ellipsoid: (longest diameter $\times$ longest perpendicular diameter $\times$ height $) / 2$. Because the change in volume was evaluated in the same patient with the same morphology of the cyst, we think this method is acceptable for this study. It was measured by K.H.K., who was not involved in the surgery.

\section{Statistical Analysis}

Continuous variables between the 2 groups were compared using the Mann-Whitney U-test, and dichotomous variables were analyzed with the chi-square test and Fisher's exact test. Statistical analyses were performed using SPSS version 25.0 software (IBM Corp.). We used 0.05 as a cutoff $\mathrm{p}$ value for statistical significance.

\section{Results}

The median age of patients was 5.5 years, and there were 40 male and 20 female patients (Table 1). The mean follow-up period was 91.3 months. Twenty-seven patients were incidentally diagnosed with an AC, whereas 19 and 14 patients showed vague and definite symptoms and signs, respectively (Table 2 ).

\section{Patients With Hydrocephalus or Papilledema}

Four patients had associated hydrocephalus, and one patient showed papilledema. Among them, 4 patients showed definite symptoms (macrocephaly, papilledema) associated with $\mathrm{AC}$, and 1 patient underwent brain CT scanning because of a head injury, and an AC with hydrocephalus was found incidentally. In the 3 patients with macrocephaly and 1 patient with papilledema, the ACs were definitely decreased in size after surgery, whereas it was slightly decreased in the asymptomatic patient. Three of the patients with hydrocephalus received microscopic fenestration, and 1 patient received endoscopic fenestration. Both SDFCs after surgery were improved without any treatment. One patient complained of newly developed third nerve palsy. In addition, 1 patient developed headache. The patient with papilledema suffered from wound swelling and CSF leakage after endoscopic fenestration and underwent cystoperitoneal $(\mathrm{CP})$ shunting.

\section{Patients Excluding Those With Hydrocephalus or Papilledema}

Because our policy is that all patients with hydrocephalus or papilledema should be operated on and the goal of this study was to evaluate the role of surgery in cases in which surgery or conservative management can be chosen, those 5 patients were excluded from the comparison of outcomes between the surgery and nonsurgery groups.

Among 33 patients who underwent surgery, 13 were found to have an incidental AC, 12 had vague symptoms of an AC, and 8 had definite symptoms of an AC (Table $3)$. Of the 22 patients who did not receive surgery, 13 were found to have an incidental AC, 7 had vague symptoms of an $\mathrm{AC}$, and 2 had definite symptoms of an AC (focal bony bulging). Forty-two ACs (76\%) were located on the left side. The ACs of the 2 patients who were observed with definite symptoms were located on the right side.

The midline shift in the surgery group versus the nonsurgery group and by symptom subgroup (mean values in incidental group: 4.5 vs $2.6 \mathrm{~mm}$; vague symptoms: 3.3 vs $2.7 \mathrm{~mm}$; and definite symptoms: $1.5 \mathrm{vs} 2.5 \mathrm{~mm}$ ) did not show any significant difference between the groups and among symptom subgrouping $(\mathrm{p}>0.05)$. The midline 
shift tended to be smaller in the definite-symptom subgroup than in the other symptom subgroups in the group of patients who underwent surgery.

Headache was relieved in almost all patients in the surgery and nonsurgery groups. In the surgery group, 3 seizure patients became seizure free with medication, which was started at the time of diagnosis and surgery, whereas in the nonsurgery group, seizure control with medication in 1 patient could not be achieved. However, 1 patient showed newly developed seizures after AC surgery. Two patients had precocious puberty; both patients underwent surgery, but the symptoms did not improve postoperatively.

The AC size difference between the surgery and nonsurgery groups was also not significant, except that the size was significantly larger $(p=0.04)$ in the vague-symptom subgroup of patients in the surgery group. No patients in the surgery group showed an increase in AC size. The size of the AC increased by more than $10 \%$ in 2 patients in the nonsurgery group, under the age of 3 years, but neither patient complained of new symptoms. Although there were more patients with significant $\mathrm{AC}$ size reductions in the surgery group than in the nonsurgery group, there was no significant difference in symptom relief between the groups.

\section{Complications}

Fourteen patients in the surgery group had complications, and complications often led to additional operations. In the case of SDFC, if the patient had a persistent or deteriorated mass effect after the operation, the SDFC was defined as a complication. Some patients had more than 2 complications concurrently. Six patients were much improved with medication or without additional operation. Eight patients needed additional operations.

\section{Cyst Fenestration}

Six of 28 patients who had cyst fenestration, microscopic or endoscopic, at their initial treatment had postoperative complications that needed additional surgery: 3 SDFCs or chronic SDHs, 1 infected SDFC with hydrocephalus, 1 CSF leak, and 1 traumatic acute SDH.

\section{Shunting Operation}

Two of 5 patients who underwent shunting surgery as their initial treatment needed additional surgery: 1 with CSF overdrainage and 1 with intractable epilepsy.

\section{Second Operation}

Traumatic acute SDH and intractable epilepsy seem to be not directly related to AC surgery. Three patients with an SDFC underwent subduroperitoneal shunting. A chronic SDH was removed through a burr hole in 1 patient. The patient with CSF leakage who had undergone microscopic fenestration at the initial surgery underwent duroplasty and $\mathrm{CP}$ shunting. Craniotomy and SDH removal were performed for a patient with an acute SDH. One patient who had undergone $\mathrm{CP}$ shunting at the initial surgery experienced overdrainage symptoms, and we removed the shunt. One patient with postshunting intractable epilepsy received a lobectomy and shunt removal with cyst fenestration (Table 4).
TABLE 1. Demographics and overall clinical features of 60 patients with large sylvian ACs

\begin{tabular}{|c|c|}
\hline Clinical Feature & No. of Cases (\%) \\
\hline \multicolumn{2}{|l|}{ Age range (mos) ${ }^{*}$} \\
\hline $0-36$ & $23(38 \%)$ \\
\hline $37-72$ & $15(25 \%)$ \\
\hline $73-258$ & $22(37 \%)$ \\
\hline \multicolumn{2}{|l|}{ Sex } \\
\hline Male & $40(67 \%)$ \\
\hline Female & $20(33 \%)$ \\
\hline \multicolumn{2}{|c|}{ Clinical presentation or reason for imaging } \\
\hline Fall & $20(33 \%)$ \\
\hline Headache & $10(16 \%)$ \\
\hline Focal bony bulging & $9(15 \%)$ \\
\hline Macrocephaly & $4(7 \%)$ \\
\hline Seizure & $4(7 \%)$ \\
\hline Traffic accident & $3(5 \%)$ \\
\hline Antenatal sonography & $3(5 \%)$ \\
\hline Precocious puberty & $2(3 \%)$ \\
\hline DD & $2(3 \%)$ \\
\hline Papilledema & $1(2 \%)$ \\
\hline Gaze palsy & $1(2 \%)$ \\
\hline Foreign body ingestion $†$ & $1(2 \%)$ \\
\hline \multicolumn{2}{|l|}{ Laterality } \\
\hline Rt & $14(23 \%)$ \\
\hline $\mathrm{Lt}$ & $46(77 \%)$ \\
\hline \multicolumn{2}{|l|}{ Location } \\
\hline Temporal & $16(26 \%)$ \\
\hline T-P-O & $1(2 \%)$ \\
\hline F-T & $38(63 \%)$ \\
\hline F-T-P & $4(7 \%)$ \\
\hline F-T-P-O & $1(2 \%)$ \\
\hline \multicolumn{2}{|l|}{ Operation (first) } \\
\hline Microscopic cyst fenestration & $24(40 \%)$ \\
\hline Endoscopic cyst fenestration & $9(15 \%)$ \\
\hline CPS & $5(8 \%)$ \\
\hline None & $22(37 \%)$ \\
\hline \multicolumn{2}{|l|}{ Size variation } \\
\hline Increased $(\geq 10 \%)$ & $2(3 \%)$ \\
\hline No change $(\uparrow<10 \%, \downarrow<30 \%)$ & $25(42 \%)$ \\
\hline Decreased slightly $(\geq 30 \%,<50 \%)$ & $11(18 \%)$ \\
\hline Decreased definitely ( $\geq 50 \%)$ & $22(37 \%)$ \\
\hline
\end{tabular}

$\mathrm{CPS}=$ cystoperitoneal shunt; $\mathrm{DD}=$ developmental delay; $\mathrm{F}-\mathrm{T}=$ frontotemporal; F-T-P = frontotemporoparietal; F-T-P-O = frontotemporoparietooccipital; T-P-O $=$ temporoparietooccipital; $\uparrow=$ increased; $\downarrow=$ decreased.

* The age of the patient at initial presentation.

† An AC was identified incidentally in the brain from a neck CT scan taken to visualize a fish bone that was stuck in the patient's throat.

\section{Subgroup Analysis According to Age Group}

We examined whether complications differed with age in the surgery group, excluding hydrocephalic or papilledema patients (Table 5). Five patients (46\%) under the age 
Kim et al.

TABLE 2. Reason for imaging study and visiting Seoul National University Children's Hospital

\begin{tabular}{ccc}
\hline $\begin{array}{c}\text { Incidental Imaging } \\
\text { Finding }(\mathrm{n}=27)\end{array}$ & $\begin{array}{c}\text { Vague } \mathrm{S} / \mathrm{Sx} \\
\text { Associated w/ ACs }(\mathrm{n}=19)\end{array}$ & $\begin{array}{c}\text { Definitely } \\
\text { FC-Related } \mathrm{S} / \mathrm{Sx}(\mathrm{n}=14)\end{array}$ \\
\hline Fall & Headache not typical of $\uparrow$ ICP & Focal bony bulging \\
\hline Traffic accident & Seizure & Macrocephaly \\
\hline Foreign body ingestion & Precocious puberty & Papilledema \\
\hline Antenatal sonography & Developmental delay & \\
\hline & Gaze palsy not caused by $\uparrow$ ICP & \\
\hline
\end{tabular}

$\mathrm{S} / \mathrm{Sx}=$ symptoms or signs.

TABLE 3. Clinical data of patients according to the initial symptoms or signs and surgical procedure, excluding the 5 patients with hydrocephalus and papilledema

\begin{tabular}{|c|c|c|c|c|c|c|}
\hline \multirow[b]{2}{*}{ Feature } & \multicolumn{3}{|c|}{ Surgery $(n=33)$} & \multicolumn{3}{|c|}{ No Surgery $(n=22)$} \\
\hline & $\begin{array}{l}\text { Incidental Imaging } \\
\text { Finding }(n=13)\end{array}$ & $\begin{array}{l}\text { Vague AC S/Sx } \\
\qquad(n=12)\end{array}$ & $\begin{array}{l}\text { Definite AC S/Sx } \\
\qquad(n=8)\end{array}$ & $\begin{array}{l}\text { Incidental Imaging } \\
\qquad(n=13)\end{array}$ & $\begin{array}{l}\text { Vague AC S/Sx } \\
\qquad(n=7)\end{array}$ & $\begin{array}{l}\text { Definite AC S/Sx } \\
\qquad(n=2)\end{array}$ \\
\hline Mean age, mos (range) & $50.3(1-98)$ & $92.6(19-258)$ & $54.6(7-126)$ & $68.3(9-215)$ & $73.3(35-110)$ & $36.5(1-72)$ \\
\hline $\mathrm{M} / \mathrm{F}$ ratio & 5.5 & 1.0 & 3 & 3.3 & 2.5 & 1.0 \\
\hline Follow-up, mos (range) & $89.9(5-235)$ & $98.5(15-305)$ & $140.9(38-228)$ & $51.7(7-148)$ & $91(26-148)$ & $87.5(61-114)$ \\
\hline \multicolumn{7}{|l|}{ Location } \\
\hline Rt/lt side & $1 / 12$ & $3 / 9$ & $3 / 5$ & $3 / 10$ & $1 / 6$ & $2 / 0$ \\
\hline Temporal & 2 & 2 & 3 & 3 & 3 & 2 \\
\hline T-P-O & 0 & 0 & 0 & 0 & 0 & 0 \\
\hline F-T & 9 & 9 & 4 & 10 & 4 & 0 \\
\hline F-T-P & 2 & 0 & 1 & 0 & 0 & 0 \\
\hline F-T-P-O & 0 & 1 & 0 & 0 & 0 & 0 \\
\hline $\mathrm{L} \times \mathrm{W} \times \mathrm{H}, \mathrm{cm}$ & $8.5 \times 5.2 \times 8.6$ & $10.1 \times 5.5 \times 9.2$ & $7.4 \times 5.0 \times 8.9$ & $7.3 \times 5.1 \times 8.4$ & $7.4 \times 5.3 \times 7.4$ & $6.7 \times 4.8 \times 5.8$ \\
\hline Midline shift, mm (range) & $4.5(0-7.9)$ & $3.3(0-6.7)$ & $1.5(0-4.0)$ & $2.6(0-6.3)$ & $2.7(0-5.5)$ & $2.5(2.3-2.7)$ \\
\hline Clinical improvement & - & $9 / 12$ & $0 / 8$ & - & $4 / 7$ & $0 / 2$ \\
\hline Focal bony bulging & & & $0 / 7$ & & & $0 / 2$ \\
\hline Macrocephaly & & & $0 / 1$ & & & \\
\hline Headache & & $6 / 6$ & & & $3 / 4$ & \\
\hline Seizure & & $3 / 3$ & & & $0 / 1$ & \\
\hline Precocious puberty & & $0 / 2$ & & & & \\
\hline DD & & $0 / 1$ & & & $1 / 1$ & \\
\hline Gaze palsy & & & & & $0 / 1$ & \\
\hline \multicolumn{7}{|l|}{ Size variation } \\
\hline Increased ( $\geq 10 \%)$ & 0 & 0 & 0 & 1 & 1 & 0 \\
\hline No change $(\uparrow<10 \%, \downarrow<30 \%)$ & 3 & 4 & 1 & 9 & 6 & 2 \\
\hline Decreased slightly $(\geq 30 \%,<50 \%)$ & 4 & 2 & 2 & 2 & 0 & 0 \\
\hline Decreased definitely ( $\geq 50 \%$ ) & 6 (1 SDFC) & 6 (1 SDFC) & 5 (1 SDFC) & 1 & 0 & 0 \\
\hline Complication & 4 & 5 & 5 & 0 & 0 & 0 \\
\hline Additional surgery & $2(1,2)^{*}$ & $2(1,6)$ & $4(1,1,2,4)$ & & & \\
\hline New ND/S & $2 \dagger$ & $1 \ddagger$ & $2 \S$ & & & \\
\hline SDFC & 2 & 1 & 1 & & & \\
\hline $\mathrm{SDH}$ & 1 & 2 & & & & \\
\hline Infection & 1 & 1 & 1 & & & \\
\hline CSF leak & & & 2 & & & \\
\hline CSF overdrainage & & & 1 & & & \\
\hline
\end{tabular}


TABLE 4. Type of first and second operations for ACs and the reasons for second surgery in 33 patients

\begin{tabular}{lcc}
\hline $\begin{array}{c}\text { Type of } \\
\text { 1st Op }\end{array}$ & Reason for 2nd Op & Type of 2nd Op \\
\hline CF & SDFC & SPS \\
\hline CF & SDFC & SPS \\
\hline EF & cSDH & Burr hole trephination \\
\hline EF & $\begin{array}{c}\text { Infection, SDFC, hydro- } \\
\text { cephalus }\end{array}$ & SPS \\
\hline CF & CSF leak & Duraplasty \& CPS \\
\hline CF & In-hospital fall, aSDH & Craniotomy \& hematoma removal \\
\hline CPS & CSF overdrainage & Shunt removal \\
\hline CPS & Intractable epilepsy & Lobectomy \& shunt removal w/ CF \\
\hline
\end{tabular}

aSDH = acute SDH; CF = craniotomy and cyst fenestration; $\mathrm{CSDH}=$ chronic $\mathrm{SDH} ; \mathrm{EF}=$ endoscopic cyst fenestration; SPS = subduroperitoneal shunt.

of 3 years and 9 patients (41\%) over the age of 3 years had complications $(\mathrm{p}=0.80)$. Four patients $(36 \%)$ under the age of 3 years and 1 patient $(5 \%)$ over the age of 3 years had an SDFC $(p=0.03)$. The mean age of patients who needed additional operations was 48.5 months (range 1-126 months), and the mean age of patients who did not need additional operations was 63.5 months (range $0-129$ months) $(p=0.39)$. The mean age of the patients who underwent 1 reoperation was 59.3 months, while that of the patients who needed 2 or more reoperations was 20.0 months $(\mathrm{p}=0.27)$. Three of 4 SDFC patients under the age of 3 years needed additional surgeries, and the patient over the age of 3 years showed improvements without additional surgery. Young patients had more SDFCs $(p=0.03)$ and a tendency toward multiple additional operations, although this difference was not statistically significant $(\mathrm{p}=$ 0.27 ). Supplementary analysis was conducted to evaluate the number of reoperations according to age group (Fig. 1). A total of 18 reoperations were performed. Eleven patients under age 3 years in the surgery group underwent 13 additional operations, whereas 22 patients over age 3 years in the same group had 5 additional operations $(\mathrm{p}<0.01)$.

No patients in the nonsurgery group received surgery during the observation period for any reason.

\section{Discussion}

The pathophysiology of ACs is not well established. In 1958, Starkman et al. ${ }^{3}$ defined an AC as a developmental anomaly located in a space separated from the subarachnoid space. In 1971, Robinson ${ }^{4}$ hypothesized that an AC is a secondary fluid collection caused by agenesis of the brain. However, it is now accepted that ACs are not caused by a loss of brain tissue, given that the volume of the brain adjacent to an AC increases after surgery. ${ }^{1}$ In addition, ACs may develop because of secondary causes such as trauma or infection. 5,6

Generally, a sylvian $\mathrm{AC}$, the most common type of $\mathrm{AC}$, is classified by Galassi type according to its size and extent. ${ }^{1}$ The natural course of ACs was studied by Al-Holou et al. ${ }^{7}$ Observation is recommended in most cases, but surgical treatment is required in some cases. Surgery can be
TABLE 5. Analysis of postoperative complications according to age group, excluding patients with hydrocephalus or papilledema

\begin{tabular}{lccc}
\hline \multirow{2}{*}{ Complication } & \multicolumn{2}{c}{ Age Group } & \\
\cline { 2 - 3 } & $<36 \operatorname{Mos}(n=11)$ & $\geq 36 \operatorname{Mos}(n=22)$ & $p$ Value \\
\hline Complication (all) & $5 / 11(46 \%)$ & $9 / 22(41 \%)$ & 0.80 \\
\hline SDFC & $4 / 11(36 \%)$ & $1 / 22(5 \%)$ & $0.03 \dagger$ \\
\hline Reoperation & $4 / 11(36 \%)$ & $4 / 22(18 \%)$ & 0.39 \\
\hline${ }^{*}$ Complications included SDFC, reoperation, neurological deficit, device \\
$\begin{array}{l}\text { exposure, CSF leak, infection, overdrainage, and SDH. } \\
\dagger p<0.05 .\end{array}$
\end{tabular}

considered in patients with symptoms such as seizures, increased ICP, neurological deficit, developmental delay, precocious puberty, and headache. The effects of surgery on these symptoms have been debated. ${ }^{8-10}$ However, surgery for ACs in patients with hydrocephalus or papilledema is generally recommended.

In some articles, large ACs (Galassi type II or III) are included in the surgical indications. ${ }^{11-14} \mathrm{~A}$ small number of researchers have claimed that all ACs have a mass effect and should be operated on to eliminate the possibility of compressing brain tissue, even if there are no symptoms. ${ }^{11,12}$ This study was conducted to ascertain whether the Galassi type III AC is an indication for surgery.

In the early days, a large $\mathrm{AC}$ itself was an indication for surgery in patients treated by our team. In the 1990s, shunting, a global trend, was the treatment of choice. Later, complications such as slit ventricle/cyst, shunt malfunction, and infections were observed after shunting. Endoscopic surgery was then later performed to overcome these problems. However, in the case of type III ACs, there was no brain parenchyma at the insertion site of the endoscope. Poor dural closure at the entry site of the endoscope and lack of brain parenchyma often cause CSF leakage. Therefore, we performed a minicraniotomy to ensure tight dural closure. Meanwhile, since 2000, some parents have

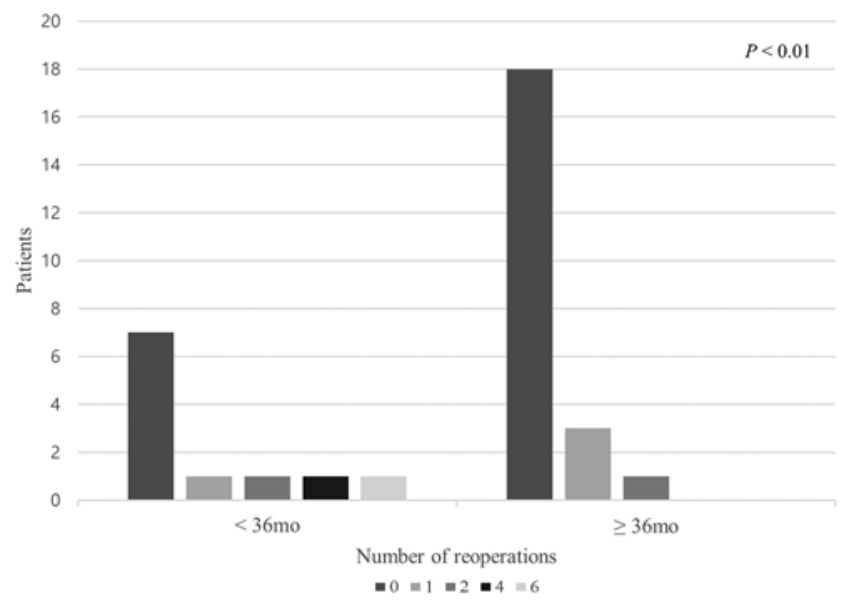

FIG. 1. Analysis of the number of reoperations according to age group of patients, $<36$ months (mo) of age or $\geq 36$ months of age, excluding those with hydrocephalus or papilledema. 
rejected surgery because their children have done well without it. Therefore, we held off on the operation and observed the patients. For years of follow-up, they continued doing fine, with or without a mild headache. Thereafter, we began suggesting two options (surgery or observation) to the parents, despite a general tendency toward surgery in cases of Galassi type III sylvian AC.

In the present study, the patient who had more obvious symptoms of AC showed less midline shift. All of the definite-symptom groups had focal bony bulging. Thus, midline shift could be relieved by the enlargement of the skull outward.

We previously reported that the size of the AC does not increase after age 3 years during follow-up. ${ }^{2}$ Similarly, in the present study, a 35-month-old patient showed an increase in $\mathrm{AC}$ size after 6 months of follow-up, but the $\mathrm{AC}$ size remained constant once the patient reached 40 months of age until the last imaging at 57 months. Another patient was diagnosed with an AC at the age of 17 months and showed an increased size at the 1-year follow-up. However, 6 months later, the AC stopped growing and remained stable in size at the last imaging follow-up at 60 months. These 2 patients did not undergo surgery because of the absence of symptoms and the transient nature of the increases in size.

We attempted to analyze whether symptom change correlated with changes in AC size after the surgical procedure. However, the analysis was not possible because there was only one patient with definite AC symptoms (the case involving papilledema) whose symptoms were relieved after surgery, even among the total surgical group cases (n $=38$ ). It appears that the change in symptoms seems to be more related to the type of symptom itself than to the change in AC size. Among the patients with definite symptoms and signs, papilledema improved, but in those with bony changes no improvement was seen.

The group with vague symptoms and signs showed a relative improvement in symptoms after surgery, but this was not different from the improvement noted in the group of patients who had been observed without surgery.

In some cases in the literature, AC surgery was effective in controlling seizures. ${ }^{15-17}$ However, in the present study, patients who had become seizure free after surgery also needed antiseizure drugs. From this perspective, it is difficult to say that AC surgery is effective in treating seizures.

According to the literature, sylvian ACs may be responsible for precocious puberty, which might be improved with surgical treatment. ${ }^{18}$ In our experience, however, ACs were not thought to be the direct cause of precocious puberty, given that there was no improvement after AC surgery. In our previous study, even in cases of suprasellar ACs, in which the influence of the $\mathrm{AC}$ on endocrine function seemed more likely, precocious puberty did not improve after surgery. ${ }^{19}$ Regarding developmental delay, neurocognitive function tests were performed in 3 patients over the age of 6 years in the present study. The cases did not show improvement after surgery. However, it is difficult to draw a conclusion on the effect of surgery due to the small number of patients. There was one patient with Duane's syndrome with gaze palsy, which was not an indication for surgery of the AC.
In this study, postoperative complications were not rare (14 of 33 patients [42\%], excluding patients with hydrocephalus or papilledema), and in 8 of the 14 patients with postoperative complications, reoperation was required. We reviewed the type of initial surgery given, indication for reoperations, and type of second surgery needed for those who required more than one operation. The majority of truly fenestration-related complications were CSF problems: SDFC with/without hemorrhage or leakage.

We analyzed whether surgical complications differed by age because of the high proportion of CSF-related postoperative complications. Excluding the patients who had hydrocephalus or papilledema, more SDFCs occurred in patients under the age of 3 years, which resulted in the need for reoperations. As benign extracerebral fluid collection occurs only in infancy, it appears that AC patients under the age of 3 years have different CSF dynamics with insufficient CSF absorption. These data support the notion that a surgical procedure for ACs in patients under the age of 3 years should be considered with greater caution.

Age younger than 3 years is well known as the time of brain development. Large ACs can compress the brain and interfere with brain development. Even if it is not evident in daily life, cyst shrinkage and brain re-expansion could promote brain development. Thus, in the present study, many patients underwent surgery, but patients under 3 years of age had many complications, whereas the neurocognitive function of patients in the nonsurgery group was good. These patients did not need neurocognitive tests for "clinical" purposes (not for research). Therefore, we needed to weigh their pros and cons regarding the influence of surgery on the neurocognitive function.

Changes in surgical indications and surgical methods over the decades during the study period are what limit this study. Despite this limitation, observation of the benign natural course in the majority of type III AC cases led us to reconsider surgical indications.

\section{Conclusions}

Large sylvian ACs, even though they occupy the whole temporal fossa with a midline shift, can be observed without any surgery if the patients do not have associated hydrocephalus or evident increased ICP, such as papilledema. Indiscreet surgical procedures for ACs could cause complications without providing benefit. Surgeons should be cautious when deciding whether to perform surgery, even in patients with Galassi type III sylvian ACs.

\section{Acknowledgments}

This work was supported in part by a National Research Foundation of Korea (NRF) grant funded by the Korean government (NRF-2018R1A5A2025964).

\section{References}

1. Galassi E, Tognetti F, Gaist G, et al. CT scan and metrizamide CT cisternography in arachnoid cysts of the middle cranial fossa: classification and pathophysiological aspects. Surg Neurol. 1982;17(5):363-369.

2. Lee JY, Kim JW, Phi JH, et al. Enlarging arachnoid cyst: a false alarm for infants. Childs Nerv Syst. 2012;28(8):12031211. 
3. Starkman SP, Brown TC, Linell EA. Cerebral arachnoid cysts. J Neuropathol Exp Neurol. 1958;17(3):484-500.

4. Robinson RG. Congenital cysts of the brain: arachnoid malformations. In: Krayenbühl H, Maspes PE, Sweet WH, eds. Progress in Neurological Surgery. Vol. 4. Karger; 1971:133174.

5. Choi J-U, Kim D-S. Pathogenesis of arachnoid cyst: congenital or traumatic? Pediatr Neurosurg. 1998;29(5):260-266.

6. Westermaier T, Schweitzer T, Ernestus RI. Arachnoid cysts. Adv Exp Med Biol. 2012;724:37-50.

7. Al-Holou WN, Yew AY, Boomsaad ZE, et al. Prevalence and natural history of arachnoid cysts in children. J Neurosurg Pediatr. 2010;5(6):578-585.

8. Arai H, Sato K, Wachi A, et al. Arachnoid cysts of the middle cranial fossa: experience with 77 patients who were treated with cystoperitoneal shunting. Neurosurgery. 1996;39(6):1108-1113.

9. Helland CA, Wester K. A population based study of intracranial arachnoid cysts: clinical and neuroimaging outcomes following surgical cyst decompression in adults. J Neurol Neurosurg Psychiatry. 2007;78(10):1129-1135.

10. Miyajima M, Arai H. Operative indication, clinical results and points on intracranial arachnoid cysts. Article in Japanese. No To Hattatsu. 2009;41(3):185-190.

11. Geissinger JD, Kohler WC, Robinson BW, Davis FM. Arachnoid cysts of the middle cranial fossa: surgical considerations. Surg Neurol. 1978;10(1):27-33.

12. Go KG, Houthoff HJ, Hartsuiker J, et al. Fluid secretion in arachnoid cysts as a clue to cerebrospinal fluid absorption at the arachnoid granulation. J Neurosurg. 1986;65(5):642-648.

13. Harsh GR IV, Edwards MS, Wilson CB. Intracranial arachnoid cysts in children. J Neurosurg. 1986;64(6):835-842.

14. LaCour F, Trevor R, Carey M. Arachnoid cyst and associated subdural hematoma. Observations on conventional roentgenographic and computerized tomographic diagnosis. Arch Neurol. 1978;35(2):84-89.
15. Koch CA, Moore JL, Voth D. Arachnoid cysts: how do postsurgical cyst size and seizure outcome correlate? Neurosurg Rev. 1998;21(1):14-22.

16. Koch CA, Voth D, Kraemer G, Schwarz M. Arachnoid cysts: does surgery improve epileptic seizures and headaches? Neurosurg Rev. 1995;18(3):173-181.

17. Sajko T, Hećimović H, Borić M, et al. Complete resolution of medically refractory temporal lobe epilepsy after arachnoid cyst fenestration. Acta Clin Croat. 2011;50(4):589-593.

18. Upadhyaya S, Nair R, Kumar V, et al. Sylvian cistern arachnoid cyst-a rare cause of precocious puberty. Pediatr Neurosurg. 2013;49(6):365-368.

19. Lee JY, Lee YA, Jung HW, et al. Long-term endocrine outcome of suprasellar arachnoid cysts. J Neurosurg Pediatr. 2017;19(6):696-702.

\section{Disclosures}

The authors report no conflict of interest concerning the materials or methods used in this study or the findings specified in this paper.

\section{Author Contributions}

Conception and design: Wang. Acquisition of data: KH Kim, Lee, Phi. Analysis and interpretation of data: KH Kim, Wang. Drafting the article: KH Kim. Critically revising the article: Wang, SK Kim, Cho. Reviewed submitted version of manuscript: all authors. Approved the final version of the manuscript on behalf of all authors: Wang. Statistical analysis: KH Kim. Administrative/technical/material support: Lee. Study supervision: Wang.

\section{Correspondence}

Kyu-Chang Wang: Seoul National University Children's Hospital, Seoul National University College of Medicine, Seoul, Republic of Korea. kcwang@snu.ac.kr. 